ADDITIONAL CONTRIBUTIONS

https://doi.org/10.1017/S1539299600008650 Published online by Cambridge University Press 


\title{
THE MICROWAVE BACKGROUND RADIATION: RECENT ADVANCES AND NEW PROBLEMS
}

\author{
G. DE ZOTTI and L. TOFFOLATTI \\ Astronomical Observatory and Department of Astronomy \\ Vicolo dell'Osservatorio, 5 \\ I-35122 Padova \\ Italy
}

\begin{abstract}
The substantially improved intensity measurements at wavelengths longward of the intensity peak of the microwave background are fully consistent with a Planck spectrum. The most precise data disagree with non-relativistic comptonization models for the large submillimeter excess observed by the NagoyaBerkeley collaboration. The interpretation of such excess as dust emission at high redshifts also faces severe difficulties. Reported anisotropies on scales of several degrees and of tens of arcsec may be contributed, at least in part, by discrete sources. Just because the best experiments at $\mathrm{cm}$ wavelengths have already got close to the source confusion limit, they also provide interesting information on the large scale distribution of radio sources. Polarimetry may be decisive in clarifying the origin of observed fluctuations.
\end{abstract}

\section{Spectrum}

Spectral measurements reported in the last three years are summarized in Table I and displayed in Fig. 1. In the range $10 \mathrm{~cm}$ to a few $\mathrm{mm}$, a key factor in improving the accuracy of the results has been a careful investigation of the atmospheric emission (Danese and Partridge 1988, and references therein). As a bonus, these investigations also led to a better understanding of the atmospheric emission itself. For example, Danese and Partridge were able to determine accurately the nonresonant $\mathrm{O}_{2}$ contribution which was previously uncertain by a factor of 2 .

There is still room for significant improvements, particularly longward of $\lambda \simeq$ $10 \mathrm{~cm}$, where Bose-Einstein-like and bremsstrahlung distortions are expected to show up. A big problem here is the accurate modelling of the emission from our own galaxy (cf. Sironi et al. 1988), as well as the subtraction of the essentially isotropic component due to unresolved discrete sources. The conventional approach (extrapolation of the low-frequency measurements of the extragalactic radio background) ignores the effect of the bimodal distribution of spectral indices of radiosources. On the other hand, the very deep source counts, now available at several radio frequencies, allow direct estimates of (or, strictly speaking, lower limits to) contributions of radio sources to the extragalactic background (see Table II).

At short wavelengths the major event was the rocket experiment by the NagoyaBerkeley collaboration (Matsumoto et al. 1988), which detected a large excess in the Wien region, similar to that reported earlier by Gush (1981). Although the authors were very careful in controlling or ruling out many possible sources of spurious signals, the results certainly need confirmation. A possible test can be provided by measurements of the dipole anisotropy at submillimeter wavelengths: 
TABLE I. Recent measurements of the MWB brightness temperature

\begin{tabular}{|c|c|c|}
\hline References & $\lambda(\mathrm{cm})$ & $T_{M W B}(K)$ \\
\hline Sironi et al 1988 & 50.0 & $2.98 \pm 0.55$ \\
\hline Levin et al 1988 & 21.3 & $2.11 \pm 0.38$ \\
\hline Sironi and Bonelli $1986 \ldots \ldots \ldots$ & 12.0 & $2.79 \pm 0.15$ \\
\hline De Amici et al $1988 \ldots \ldots \ldots \ldots$ & 8.1 & $2.59 \pm 0.13$ \\
\hline Mandolesi et al 1986b . ......... & 6.3 & $2.70 \pm 0.07$ \\
\hline Kogut et al 1988 & 3.0 & $2.61 \pm 0.06$ \\
\hline Johnson and Wilkinson $1987 \ldots$ & 1.2 & $2.783 \pm 0.025$ \\
\hline De Amici et al (1985) .......... & 0.909 & $2.81 \pm 0.12$ \\
\hline Bersanelli (Smoot et al 1987) .. & 0.333 & $2.60 \pm 0.10$ \\
\hline Peterson et al $1985 \ldots \ldots \ldots \ldots$ & 0.351 & $2.80 \pm 0.16$ \\
\hline Meyer and Jura 1985 & 0.264 & $2.70 \pm 0.04$ \\
\hline Crane et al. $1988 \quad \ldots \ldots \ldots \ldots$ & 0.264 & $2.796_{-0.040}^{+0.018}$ \\
\hline Peterson et al $1985 \ldots \ldots \ldots \ldots$. & 0.198 & $2.95_{-0.12}^{+0.11}$ \\
\hline Peterson et al $1985 \ldots \ldots \ldots \ldots$ & 0.148 & $2.92 \pm 0.10$ \\
\hline Meyer and Jura $1985 \ldots \ldots \ldots$ & 0.132 & $2.76 \pm 0.20$ \\
\hline Palazzi et al. 1988 & 0.132 & $2.67 \pm 0.10$ \\
\hline Matsumoto et al $1988 \ldots \ldots \ldots$ & 0.116 & $2.799 \pm 0.018$ \\
\hline Peterson et al $1985 \ldots \ldots \ldots \ldots$ & 0.114 & $2.65_{-0.10}^{+0.09}$ \\
\hline Peterson et al $1985 \ldots \ldots \ldots \ldots$ & 0.100 & $2.55_{-0.18}^{+0.14}$ \\
\hline Matsumoto et al $1988 \ldots \ldots \ldots$ & 0.0709 & $2.955 \pm 0.017$ \\
\hline Matsumoto et al 1988 ........ & 0.0481 & $3.175 \pm 0.027$ \\
\hline
\end{tabular}

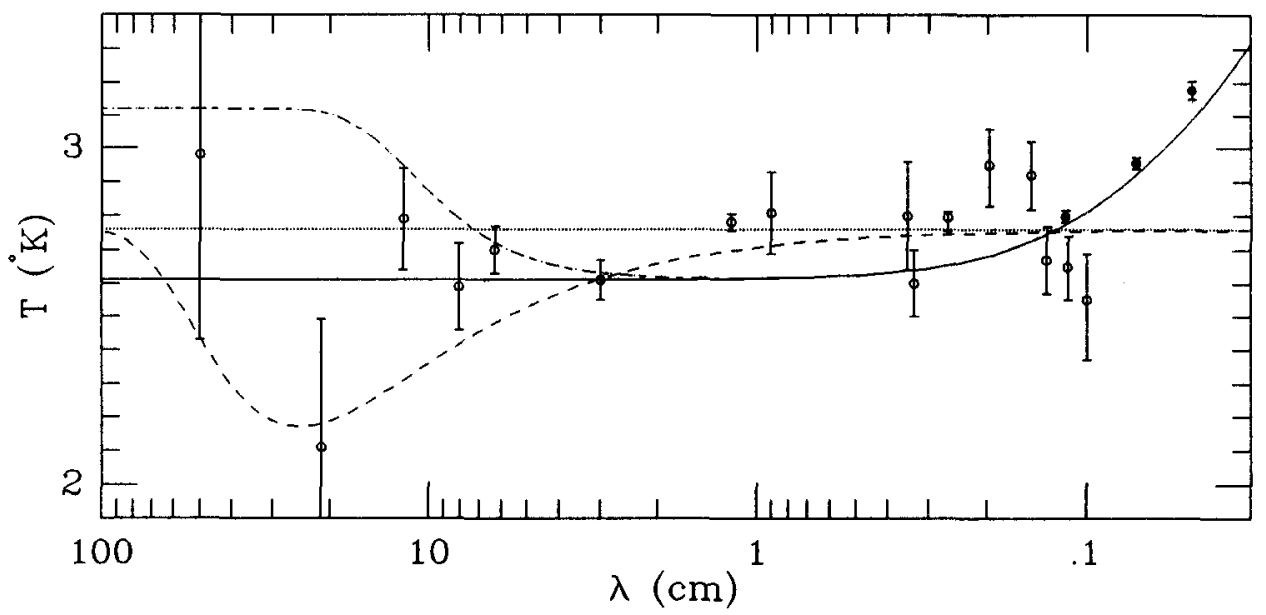

Figure 1. Comparison of the data in Table I with potential distortions of the MWB spectrum: non-relativistic comptonization with $y=0.025$ (solid line); bremsstrahlung distortion for the same value of $y, z_{\text {heating }}=10^{4}$, and $\Omega_{b a r y o n}=1$ (dot-dashed line); Bose-Einstein distortion with chemical potential $\mu_{0}=1 \times 10^{-2}$ and $\Omega_{\text {baryon }}=0.1$.

in the presence of the reported rise of the brightness temperature, the ComptonGetting effect would lead to a decrease of the dipole amplitude (Danese and De Zotti 1981). This expectation is not borne out by the measurements of Halpern et 
TABLE II. Contributions of discrete sources to the sky brightness temperature

\begin{tabular}{ccc}
\hline$\nu(G H z)$ & $\lambda(\mathrm{cm})$ & $T_{\text {sources }}(K)$ \\
\hline 0.178 & 168.5 & 21.8 \\
0.408 & 73.5 & 2.585 \\
0.6 & 50.0 & .88 \\
0.8 & 37.5 & .40 \\
1.4 & 21.4 & .094 \\
2.5 & 12.0 & .020 \\
\hline
\end{tabular}

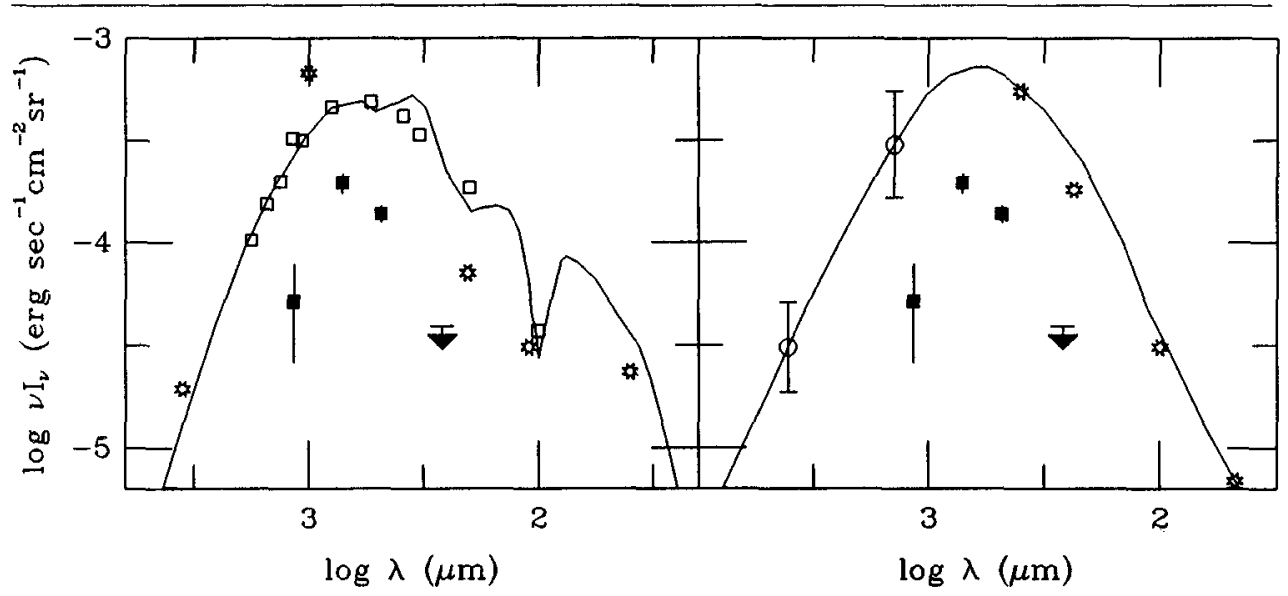

Figure 2. Comparison of the Matsumoto et al. (1988) excess (filled squares and arrow) with observed shapes of dust emission spectra. The panel on the left hand side shows the shape of the spectrum of the 3-kpc ring in NGC 1068 (open squares) and of regions of massive star formation in our Galaxy (stars); for references on the data and on the model (solid line), see Rowan-Robinson (1987). The data in other panel refer to the isolated dark cloud L 810 (Chini et al. 1986). Dust emission spectra have been arbitrarily redshifted and scaled in flux to ease the comparison with the data by Matsumoto et al. (1988).

al. (1988), although the disagreement is only at the $2 \sigma$ level.

If the sub-mm excess is real, two possible explanations come immediately to mind, and indeed have already been discussed by Hayakawa et al. (1987), Smoot et al. (1988), and others.

A first possibility is comptonization by non-relativistic electrons. Since the excess energy is $\Delta \epsilon / \epsilon \simeq 10 \%$, the comptonization parameter is $y \simeq \frac{1}{4}(\Delta \epsilon / \epsilon) \simeq 0.025$. The corresponding comptonized spectrum is shown in Fig. 1. To obtain a good fit to the Nagoya-Berkeley data we would actually need a slightly larger comptonization parameter $(y \simeq 0.028$, Hayakawa et al. 1987). But a larger $y$ would further enhance the disagreement with the two most accurate results at longer wavelengths.

Kawasaki and Sato (1987) claim that a good fit may be obtained with comptonization of photons produced by radiative decay of massive weakly interacting particles having appropriate mass, lifetime, and number density. On the other hand, they apparently don't use the right electron temperature as a function of time. For example, after the end of the decay process, their formula yields $T_{e} \simeq T_{R}(1+y)$, 
while the correct result is $T_{e} \simeq T_{R}(1+5.4 y)$. In addition, particle decay is further severely constrained by consideration of the stability of late stages of stellar evolution.

The difficulty with the data at $\lambda \geq 0.264 \mathrm{~cm}$ is somewhat relieved in the case of comptonization by relativistic electrons (Wright 1979), such as those invoked by some models for a bremsstrahlung origin of the $\mathrm{X}$-ray background (Barcons and Fabian 1988). However, a spectrum of this kind does not provide a good fit to the Nagoya-Berkeley data (Hayakawa et al. 1987); in particular, it tends to conflict with the tight upper limit at $0.26 \mathrm{~mm}$.

An alternative possibility (Hayakawa et al. 1987; Hogan and Bond 1988) is reradiation from dust heated by pregalactic stars or by massive starbursts during early phases of galaxy evolution. This picture, in addition to energetic problems (Lacey and Field 1988), has serious difficulties in accounting for the shape of the observed excess. After subtraction of a blackbody at $T=2.762 \pm 0.013 \mathrm{~K}$ (the weighted mean of data at $\lambda \geq 0.264 \mathrm{~mm}$ listed in Table I) we end up with a remarkably narrow residual spectrum, well fitted by a single temperature dust emission. As illustrated by Fig. 2, the observed dust emission spectra in a variety of astrophysical settings are substantially broader: very special conditions are apparently required for the dust to be strictly isothermal. In addition, it appears to be difficult to avoid the further smearing out due to the redshift distribution of the emitting dust.

\section{Isotropy}

\subsection{LARGE ANGULAR SCALES}

The dipole anisotropy is now measured with great accuracy. The data from radiometers carried aloft by balloons, by the Berkeley group (Lubin et al. 1985) and by the Princeton group (Fixen et al. 1983), each covering $\simeq 85 \%$ of the sky, have been combined into a map which is better connected and has a sky coverage increased to $\simeq 90 \%$ (Lubin and Villela 1986). The average dipole amplitude is $\Delta T=3.26 \pm 0.23 \mathrm{mK}$, and the average solar velocity direction is $\alpha=11^{h} .25 \pm 0^{h} .15$, $\delta=-5^{\circ} .6 \pm 2^{\circ} .0$. Both results are in excellent agreement with those of the Relict experiment aboard the Prognoz 9 satellite (Strukov et al. 1987) which has scanned the whole celestial sphere: $\Delta T=3.16 \pm 0.12 m K, \alpha=11^{h} .28 \pm 0^{h} .16, \delta=-7^{\circ} .5 \pm 2^{\circ} .5$.

As is well known, the microwave dipole is a crucial benchmark for studies of large-scale streaming motions (see, e.g., Lynden-Bell et al. 1988).

No signal was found for higher harmonics. The quadrupole limits of both balloon experiments are $\Delta T / T \leq 7 \times 10^{-5}$. An harmonic analysis of Relict data yields $\Delta T / T \leq 3 \times 10^{-5}$. Even tighter constraints are obtained from the variance analysis for specific fluctuation spectra (Strukov et al. 1988); for example, for a Zeldovich spectrum $(\Delta T / T)_{\text {quadrupole }} \leq 1.6 \times 10^{-5}$ at the $95 \%$ confidence.

\subsection{SMALL ANGULAR SCALES}

Recent results on anisotropies at intermediate and small angular scales are listed in Table III.

Significant fluctuations with an observed standard deviation of $3.7 \times 10^{-5}$ on scales of $8^{\circ}-10^{\circ}$ have been reported by the Jodrell-IAC group (Davies et al. 1987), at $\lambda \simeq 2.87 \mathrm{~cm}$. While recognizing that structure in the radio continuum emission from our galaxy may contribute appreciably, the authors argue that a substantial 
TABLE III. Small scale anisotropies

\begin{tabular}{lccc}
\hline Reference & $\lambda(\mathrm{cm})$ & $\theta$ & $\frac{\Delta T}{T}$ \\
\hline $\begin{array}{l}\text { Davies et al. }(1987) \\
\text { Klypin et al. (1987) }\end{array}$ & 3 & $8^{\circ}$ & $3.7 \times 10^{-5}$ \\
Mandolesi et al. (1986a) & 0.8 & $6^{\circ}$ & $\leq 5.6 \times 10^{-6}$ \\
& 3. & $2^{\circ}-6^{\circ}$ & $\leq 5-7 \times 10^{-4}$ \\
Uson and Wilkinson (1985) & 1.5 & $4^{\prime} .5$ & $\leq 2 \times 10^{-5}$ \\
Readhead et al. (1988) & 1.5 & $7^{\prime}$ & $\leq 1.5 \times 10^{-5}$ \\
Martin and Partridge (1988) & 6 & $18^{\prime \prime}-80^{\prime \prime}$ & $(1.7 \pm 0.5) \times 10^{-4}$ \\
Martin and Partridge (1988) & 6 & $36^{\prime \prime}-160^{\prime \prime}$ & $(1.3 \pm 0.2) \times 10^{-4}$ \\
Hogan and Partridge (1988) & 2 & $18^{\prime \prime}$ & $(2 \pm 1) \times 10^{-4}$ \\
Fomalont et al. $(1988)$ & 6 & $12^{\prime \prime}$ & $\leq 8.5 \times 10^{-4}$ \\
Fomalont et al. $(1988)$ & 6 & $18^{\prime \prime}$ & $\leq 1.2 \times 10^{-4}$ \\
Fomalont et al. (1988) & 6 & $30^{\prime \prime}$ & $\leq 0.8 \times 10^{-4}$ \\
Fomalont et al. $(1988)$ & 6 & $60^{\prime \prime}$ & $\leq 0.6 \times 10^{-4}$ \\
\hline
\end{tabular}

part of the signal is probably intrinsic. Fluctuations of similar amplitude on an angular scale of $6^{\circ}$ were previously observed by Melchiorri et al. (1981) at sub-mm wavelengths; again, the interpretation of the result depends on an uncertain correction for the galactic contribution. More recently, the Jodrell-IAC group (Watson et al. 1988) has obtained new results on an angular $\simeq 5^{\circ}$. They were able to identify all features in the scan which have temperature excesses $\Delta T / T>3 \times 10^{-5}$ with beam-diluted known sources, with the exception of a peak in the direction $\alpha \simeq 14^{h} 40^{m}, \delta \simeq 40^{\circ}$; they note that this is the direction of the Bootes void as well as of an unusual string of galaxies. No signal of this amplitude has been reported by the Relict experiment, operating at $\lambda=8 \mathrm{~mm}$.

High sensitivity observations by several groups have led to remarkably tight upper limits on arcminute scales. At the same time, improved methods have been developed to compare the experimental results with theoretical predictions (Bond and Efstathiou 1987; Vittorio and Juskiewicz 1987). The analysis of Lasenby and Kaiser (1988) has shown that the upper limit originally reported by Uson and Wilkinson (1985), and quoted in Table III, should be revised upwards by a factor $\geq 2$.

On still smaller angular scales, around or below $1^{\prime}$, a signal has definitely been detected. Its interpretation, however, is still controversial. While Martin and Partridge (1988) argue that discrete sources cannot explain all the variance they see, Fomalont et al. (1988) come to the opposite conclusion. Franceschini et al. (1988) confirm that the counts of known classes of radio sources fall short by a factor $\simeq 3$ to account for the fluctuation amplitude measured by Martin and Partridge. On the other hand, the results of the VLA survey at $\lambda \simeq 20 \mathrm{~cm}$ by Mitchell and Condon (1985) may already suggest that true counts are substantially higher than predicted by current models, and could saturate the observed sky variance. The discrete source contribution to fluctuations could be further enhanced by clustering.

Careful reviews of the quantitative predictions for anisotropies of the microwave background for a wide variety of theories of the formation of structure in the universe have recently been presented by Kaiser and Silk (1986), Bond (1988), and Hogan and Bond (1988). 


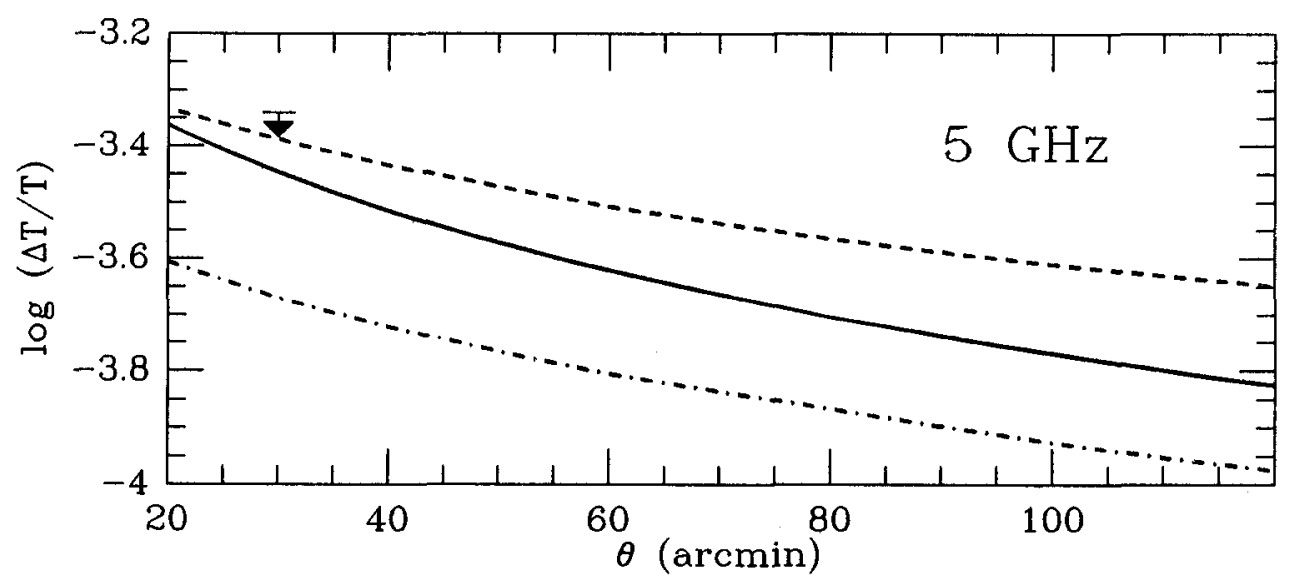

Figure 3. Contribution of discrete sources to temperature fluctuations, as a function of the angular scale. The solid line refers to a Poisson distribution of sources (Franceschini et al. 1988). The dashed and the dot-dashed lines show the additional contributions due to clustering described by a power-law $\xi(r)$ (see text) with $r_{0}=40 M p c$ and $r_{0}=20 M p c\left(H_{0}=50\right)$, respectively, and $r_{\max }=2 r_{0}$. The upper limit is from Lasenby and Davies (1983).

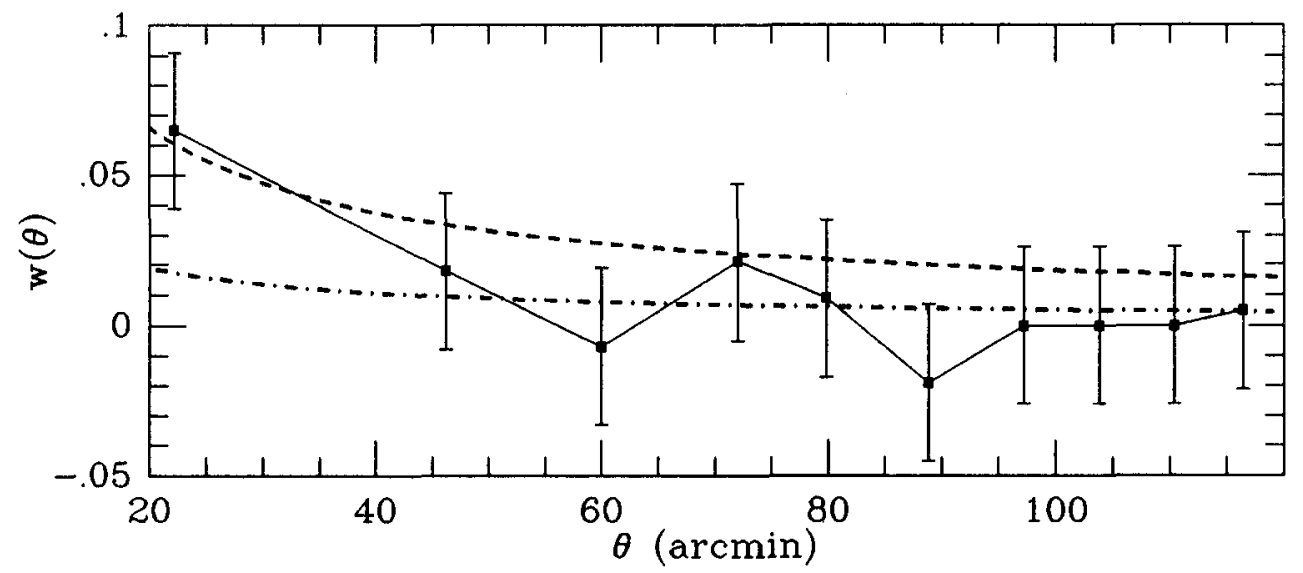

Figure 4. Comparison of the angular correlation functions corresponding to the same choices for $\xi(r)$ as in Fig. (3) with the data of Langston (1988).

\subsection{SUNYAEV-ZELDOVICH EFFECT}

The Sunyaev-Zeldovich dips on scales of a few arcmin, in the directions of three rich clusters of galaxies were the first small scale anisotropies for which detection at a high level of significance has been reported (Birkinshaw 1987). On the other hand, many years of experimental work have revealed several astrophysical and instrumental effects that may distort the results (cf. Partridge et al. 1987 and references therein); a full exploitation of the rich astrophysical information provided by the $\mathrm{S}-\mathrm{Z}$ effect is probably still premature. 


\subsection{FLUCTUATIONS DUE TO DISCRETE SOURCES}

As already mentioned, small scale temperature fluctuations due to the non-uniform space distribution of discrete sources may seriously hinder searches for primordial anisotropies, except for a relatively narrow frequency range (Franceschini et al. 1988).

On the other hand, fluctuations due to sources are themselves interesting quantities to measure. For example, they may be informative on the large-scale distribution of radio-sources, which, in turn, may reflect the large-scale distribution of matter in general. In Fig. 3 we compare the Poisson contributions to $\Delta T / T$ with the additional fluctuations expected if radio-sources have a power-law twopoint correlation function, $\left[\xi(r)=\left(r / r_{o}\right)^{-1.8}\right]$, cut down at the radius $r_{\max }$. For $r_{0} \geq 30-40 M p c$ the fluctuations are dominated by the effect of clustering, and the upper limit set by Lasenby and Davies (1983) already implies that $r_{0}$ cannot be much larger than $40 \mathrm{Mpc}$. This constraint is significantly stronger than that obtained from the study of the two-point angular correlation function of sources in the MG II survey (Langston 1988; see Fig. 4).

\section{Polarization}

Until recently, both observational and theoretical studies dealt with polarization on large angular scales, introduced by anisotropic expansion (Tolman 1985, and references therein).

As first pointed out by Kaiser (1983), however, a significant degree of polarization is associated with small scale anisotropies induced by adiabatic perturbations. Detailed studies (Milaneschi and Valdarnini 1986; Bond and Efstathiou 1987) have shown that, for a variety of models for primordial perturbations, the predicted $\Delta T_{p} / T$ range from $\simeq 2 \%$ to $\simeq 10 \%$ of intensity fluctuations. The ratio $\left(\Delta T_{p} / T\right) /(\Delta T / T)$ peaks at small angular scales. As pointed out by Milaneschi and Valdarnini (1986), this can be easily understood since long wavelength perturbations contribute to temperature anisotropies through potential fluctuations, but there are not enough scatterings for them to generate polarization (in dealing with very large perturbations, recombination can be treated as instantaneous). Similarly, $\left(\Delta T_{p} / T\right) /(\Delta T / T)$ decreases as the spectral index $n$ of the primordial perturbation spectrum $\left(\delta^{2} \propto k^{n}\right)$ decreases, since the relative power on large scales increases.

Polarimetry may thus be decisive in determining the origin of anisotropies, in the presence of confusion from faint sources.

Limits on linear and circular polarization (Stokes parameters Q, U, and V) for scales $18^{\prime \prime} \leq \theta \leq 160$ " were recently reported by Partridge et al. (1988). They range from $\left(\Delta T_{p} / T\right) \leq 4 \times 10^{-5}$ to $\left(\Delta T_{p} / T\right) \leq 2.2 \times 10^{-4}$, and are about three times lower than upper limits on intensity fluctuations set on the same angular scales.

Acknowledgements. We are indebted to L. Danese and A. Franceschini for many useful discussions. Work supported in part by MPI and CNR (through GNA and PSN).

\section{References}

Barcons, X., and Fabian, A.C. 1988, M.N.R.A.S., $230,139$. 
Birkinshaw, M. 1987, in Observational Cosmology, A. Hewitt et al. (eds.), p. 83.

Bond, J.R. 1988, in Large Scale Structures of the Universe, Kluwer, p. 93.

Bond, J.R., and Efstathiou, G. 1987, M.N.R.A.S., 226, 655.

Chini, R., et al. 1986, in Light on Dark Matter, F.P. Israel (ed.), Reidel, p. 29.

Crane, P., et al. 1988, in Proc. Third ESO/CERN Symposium, in press.

Danese, L., and De Zotti, G. 1981, Astr. Ap., 94, L33.

Danese, L., and Partridge, R.B. 1988, Ap. J., submitted.

Davies, R.D., et al. 1987, Nature, 326, 462.

De Amici, G., et al. $1988, A p$. J., 329, 556 .

De Amici, G., Smoot, G.F., Friedman, S.D., and Witebsky, C. 1985, Ap. J., 298, 710.

Fixen, D.J., Cheng, E.S., and Wilkinson, D.T. 1983, Phys. Rev. Letters, 50, 620.

Fomalont, E.B., et al. $1988, A . J ., 96,1187$.

Franceschini, A., Toffolatti, L. Danese, L., and De Zotti, G. 1988, Ap. J., submitted.

Gush, H.P. 1981, Phys. Rev. Letters, 47, 745.

Halpern, M., et al. $1988, A p$. J., $332,596$.

Hayakawa, S., et al. 1988, Publ. Astron. Soc. Japan, $39,941$.

Hogan, C.J., and Bond, J.R. 1988, in The Post-Recombination Universe, N. Kaiser and A.N. Lasenby (eds.), Kluwer, p. 141.

Hogan, C., and Partridge, R.B. 1988, preprint.

Johnson, D.G., and Wilkinson, D.T. 1987, Ap. J. Letters, 313, L1.

Kaiser, N. 1983, M.N.R.A.S., $202,1169$.

Kaiser, N., and Silk, J. 1986, Nature, 324, 529.

Kawasaki, M., and Sato, K. 1987, Publ. Astr. Soc. Japan, 39, 837.

Klypin, A.A., et al. 1987, Pis'ma Astron. Zh., 13, 259 [Sov. Astron. Letters, 13, 194]

Kogut, A., et al. $1988, A p$. $J, 325,1$.

Lacey, C.G., and Field, G.B. 1988, Ap. J. Letters, 330, L1.

Langston, G. 1988 in Large Scale Structure and Motions in the Universe, in press.

Lasenby, A.N., and Davies, R.D. 1983, M.N.R.A.S., 203, 1137.

Lasenby, A.N., and Kaiser, N. 1988, preprint.

Levin, S.M. et al. 1988 Ap. J., in press.

Lubin, P., Villela, T. 1986, in Galaxy Distances and Deviations from Universal Expansion, B.F. Madore and R.B. Tully (eds.), Reidel, P. 169.

Lubin, P., Villela, T., Epstein, G., and Smoot, G.F. 1985, Ap. J. Letters, 298, L1.

Lynden-Bell, D., et al. 1988, Ap. J., 326, 19.

Mandolesi, N., et al. 1986a, Nature, 319,751.

Mandolesi, N., et al. $1986 \mathrm{~b}, A p . J ., 310,561$.

Martin, H.M., and Partridge, R.B. 1988, Ap. J., 324, 794.

Matsumoto, T., et al. $1988, A p$. J. 329,567 .

Melchiorri, F., et al. $1981, A p$. J. Letters, $250, \mathrm{~L} 1$.

Meyer, D.M., and Jura, M. 1985, Ap. J., 297, 119.

Milaneschi, $\mathrm{E}$., and Valdarnini, $\mathrm{R}$. 1986, Astr. Ap., 162, 5.

Mitchell, K.J., and Condon, J.J. 1985, A. J., 90, 1957.

Palazzi, et al. 1988, in Proc. Third ESO/CERN Symposium, in press.

Partridge, R.B., Nowakowski, J., and Martin, H.M. 1988, Nature, 331, 146.

Partridge, R.B.' Perley, R.A., Mandolesi, N., and Delpino, F 1987, Ap. J, 317, 112.

Peterson, J.B., Richards, P.L., and Timusk, T. 1985, Phys. Rev. Letters, 55, 332.

Readhead, A.C.S., et al. 1988, in Large Scale Structures of the Universe, Kluwer, p. 37.

Rowan-Robinson, M. 1987, in Star Formation in Galaxies, C.J. Lonsdale (ed.), p. 133.

Sironi, G., and Bonelli, G. 1986, Ap. J., 311, 418.

Sironi, G., et al. $1988, A p$. J., submitted.

Smoot, G.F., et al. 1987, Ap. J. Letters, 317, L45.

Smoot, G.F., et al. 1988, Ap. J., 331, 653 .

Strukov, I.A., et al. 1987, Pis'ma Astron. Zh., 13, 163 [Sov. Astron. Letters, 13, 65]

Tolman, B.W. 1985, Ap. J., 290, 1.

Uson, J.M., and Wilkinson, D.T. 1985, Nature, 312, 427.

Vittorio, N., and Juskiewicz, R. 1987, Ap. J. Letters, 314, L29.

Watson, R.A., et al. 1988, in Large Scale Structure and Motions in the Universe, in press.

Wright, E.L. $1979, A p . J ., \mathbf{2 3 2}, 348$. 